\title{
La procédure de contrôle URSSAF : un système à parfaire
}

\section{François Taquet}

\section{(2) OpenEdition}

1 Journals

\section{Édition électronique}

URL : https://journals.openedition.org/rdctss/1203

DOI : $10.4000 /$ rdctss. 1203

ISSN : 2262-9815

Éditeur

Centre de droit comparé du travail et de la sécurité sociale

\section{Édition imprimée}

Date de publication : 1 avril 2020

Pagination : 92-101

ISSN : 2117-4350

\section{Référence électronique}

François Taquet, "La procédure de contrôle URSSAF : un système à parfaire », Revue de droit comparé du travail et de la sécurité sociale [En ligne], 1 | 2020, mis en ligne le 01 novembre 2021, consulté le 11 novembre 2021. URL : http://journals.openedition.org/rdctss/1203 ; DOI : https://doi.org/10.4000/ rdctss. 1203

\section{(c) (i) $\odot$}

Revue de droit comparé du travail et de la sécurité sociale est mise à disposition selon les termes de la Licence Creative Commons Attribution - Pas d'Utilisation Commerciale - Pas de Modification 4.0 International. 


\title{
LA PROCÉDURE DE CONTRÔLE URSSAF : UN SYSTÈME À PARFAIRE
}

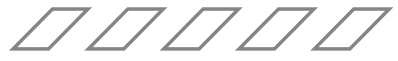

\section{RÉSUMÉ}

En France, les Unions de recouvrement des cotisations de Sécurité sociale (URSSAF) ont notamment pour mission de collecter les cotisations et contributions sociales et d'effectuer des contrôles auprès des entreprises afin de vérifier la bonne application de la réglementation. Nul ne contestera dans un système déclaratif, l'existence de contrôles. Encore faut-il que ceux-ci se déroulent dans un climat apaisé et que le cotisant puisse bénéficier de garanties réelles. Est-ce le cas ? La suite de notre propos nous amènera à formuler un certain nombre de propositions afin d'améliorer les relations URSSAF/ Entreprises.

MOTS CLÉS : Sécurité sociale, contrôle, recouvrement.

\begin{abstract}
In France, the Unions for the recouvery of Social Security Contributions (URSSAF) are notably responsible for collecting social security contributions and contributions and carrying out inspections of companies in order to verify the proper application of the regulations. No one will contest in a declarative system the existence of controls. It is still necessary that these take place in a peaceful climate and that the contributor can benefit from real guarantees. Is that the case? The rest of our remarks will lead us to formulate a number of proposals in order to improve URSSAF / Business relations.
\end{abstract}

KEYWORDS : Social Security, Control, Recovery. 


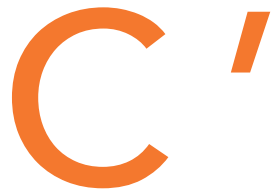

est en 1945 qu'Alexandre Parodi, alors Ministre du travail, a confié à Pierre Laroque ${ }^{1}$ la mission d'élaborer une réforme " ambitieuse " en matière de protection sociale. Le régime général de la Sécurité sociale, ainsi créé par les ordonnances des 4 et 19 octobre 1945, reposait sur les principes d'universalité, d'unicité et de solidarité nationale. Chaque branche était compétente en matière de prestations et de recouvrement des cotisations; pour simplifier le système, la loi de finances n52-401 du 14 avril 1952 avait prévu la possibilité que les Caisses de Sécurité sociale organisent un service commun de recouvrement des cotisations sociales. Toutefois, c'est avec le décret n60-452 du 12 mai 1960 que la création des URSSAF a été rendue obligatoire.

Ces organismes de droit privé ${ }^{2}$ chargés d'une mission de service public ont notamment pour objectifs de collecter les cotisations et contributions sociales, afin de les redistribuer auprès des caisses prestataires, et d'effectuer des contrôles auprès des entreprises pour vérifier la bonne application de la réglementation ${ }^{3}$. Selon les statistiques, les recettes encaissées par les URSSAF seraient aujourd'hui plus importantes que celles collectées par l'Etat au titre des impôts ou de la TVA 4 . Quant aux contrôles, ils se résument en quelques données : 124000 actions en 2018, avec plus de 50\% des cotisations déclarées vérifiées ces quatre dernières années, un contrôle sur deux détectant une irrégularité 5 . C'est dire que ces organismes sont considérés comme essentiels pour le financement de la protection sociale et, dans le cadre du contrôle, estimés comme très performants. Cependant, la tâche n'est guère aisée pour le législateur, tiraillé entre la nécessité de financer rapidement un régime qui réclame de plus en plus de moyens, et l'obligation d'offrir à tout cotisant un cadre juridique respectueux du dialogue et de la procédure contradictoire. Force est de constater que les URSSAF n'ont pas toujours bonne presse, d'aucuns pointant du doigt certains abus ou des méthodes de contrôle contestables, au milieu d'une législation

1 Souvent appelé le «père » de la sécurité sociale de 1945, Pierre Laroque (1907-1997) fut Directeur Général des assurances sociales puis de la Sécurité sociale de 1944 à 1951.

2 Dont 22 Urssaf régionales, 1 Caisse Commune de Sécurité Sociale (CCSS) en Lozère, 1 Caisse de Sécurité Sociale à Mayotte, 4 Caisses Générales de Sécurité Sociale (CGSS) dans les Départements d'Outre-mer. Chaque URSSAF est administrée par un conseil d'administration de 20 membres (art. L 213-2 du Code de la Sécurité sociale, ci-après CSS).

3 CSS, art. L 213-1.

$4516 \mathrm{Mds}$ d'Euros d'encaissements pour les URSSAF, contre $414 \mathrm{Mds}$ d'Euros de recettes fiscales : www.acoss.fr

5 Soit 834 millions d'Euros de régularisations en 2018. Pour les entreprises de plus de 250 salariés, 9 contrôles sur 10 aboutissent à une régularisation (voir le rapport d'activité ACOSS 2018). 
sociale de plus en plus complexe ${ }^{6}$. Certes, quelques décrets sont intervenus pour tenter d'améliorer les relations URSSAF/usagers ${ }^{7}$. Mais est-ce suffisant?

Cette étude propose des pistes de réflexion critique sur le cadre de l'avis de contrôle (I), de la vérification (II), et du contentieux (III).

\section{I - UNE LÉGISLATION PERFECTIBLE SUR L'AVIS DE CONTRÔLE}

Le Décret n99-434 du 28 mai 1999 a rendu obligatoire l'envoi d'un avis de contrôle avant toute vérification, sauf en cas de travail clandestin. Deux idées étaient à l'origine de cet apport : tout d'abord, I'article L47 du Livre des Procédures Fiscales (ci-après LPF) obligeait les agents des impôts, avant toute vérification, à envoyer au contribuable un avis de vérification mentionnant les années soumises à contrôle, et à lui proposer la possibilité de se faire assister par un conseil de son choix ${ }^{8}$. II convient ici de souligner l'attractivité du droit fiscal sur le droit de la sécurité sociale. Par ailleurs, un groupe de travail chargé par le Gouvernement en 1987 d'étudier les moyens susceptibles d'améliorer les relations entre les usagers et les URSSAF avait proposé l'envoi obligatoire d'un avis de passage, sauf dans le cas de travail clandestin. II fallut toutefois plus de dix ans pour que cette idée, somme toute simple, se concrétise?.

Dans sa version actuelle, l'article R 243-59 I du Code de la Sécurité sociale (ci-après CSS) précise que «tout contrôle effectué en application de l'article L. 243-7 est précédé, au moins quinze jours avant la date de la première visite de l'agent chargé du contrôle, de l'envoi par l'organisme effectuant le contrôle des cotisations et contributions de sécurité sociale d'un avis de contrôle. Toutefois, l'organisme n'est pas tenu à cet envoi dans le cas où le contrôle est effectué pour rechercher des infractions aux interdictions mentionnées à l'article L. 8221-1 du Code du travail. Dans ce dernier cas, si l'organisme entend poursuivre le contrôle sur d'autres points de la réglementation, un avis de contrôle est envoyé selon les modalités définies au premier alinéa ». Les termes sont ici très clairs : hormis dans le cadre du travail dissimulé, I'URSSAF doit obligatoirement envoyer avant le contrôle un avis de passage, sans que soit exigée la preuve d'un préjudice pour le cotisant ${ }^{10}$.

6 Voir notamment : https://www.lecerclelafay.fr/ (qui vise à améliorer les relations URSSAF/ Entreprises); Rapport parlementaire Fouquet « Cotisations sociales : stabiliser la norme, sécuriser les relations avec les URSSAF et prévenir les abus », La Documentation Française, 2008 ; Rapport parlementaire Gérard-Goua "Pour un nouveau mode de relations URSSAF/entreprises " (avril 2015); les propositions de loi $n^{\circ} 533$ de D. Jacquat (1995), n¹693 de F.-X. Villain (2004), $\mathrm{n}^{\circ} 263$ de J. Legendre (2005) et $n^{\circ} 572$ de P. Gruny (2019).

7 Décret n99-434 du 28 mai 1999 ; Décret n²007-546 du 11 avril 2007 ; Décret n²016-941 du 8 juillet 2016.

8 Le vérificateur devait également envoyer ou remettre à l'intéressé la " Charte des droits et obligations du contribuable vérifié » (LPF, art. L10 al. 4).

9 A l'époque, cette avancée ne constituait qu'un affichage puisqu'elle ne prévoyait ni le délai devant s'écouler entre l'envoi de cet avis et le contrôle lui-même, ni le contenu du document, ni l'obligation de mentionner la possibilité de se faire assister d'un conseil. Voir F. Taquet, «La réforme inachevée des règles relatives au contrôle URSSAF », Droit social, 2000, p. 1100.

10 Cour d'appel de Paris, pôle 6, ch. 12, 5 juin 2014, n¹1/07373. En cas de litige, c'est à l'URSSAF d'apporter la preuve de l'envoi de ce document. 


\section{LA PROCÉDURE DE CONTRÔLE URSSAF}

Quant à la notion de travail dissimulé11, il convient de se référer au Code du travail. Rappelons que cette définition est particulièrement large puisque le fait de mentionner, sur le bulletin de paie, un nombre d'heures de travail inférieur à celui réellement effectué, constitue une dissimulation d'emploi salarié ${ }^{12}$. II est cependant clair que cette exemption d'avis de contrôle ne saurait jouer que si le contrôle a été engagé afin de rechercher des infractions dans le cadre du travail dissimulé ${ }^{3}$. Si le contrôle porte sur des infractions au travail dissimulé, mais également sur un contrôle normal d'assiette, un avis de passage devrait être envoyé pour cette dernière vérification ${ }^{14}$.

L'obligation étant posée, il convient désormais de s'intéresser au contenu du document. À y regarder de près, les mentions qui doivent être prévues par l'avis ne sont pas nombreuses.

En effet, le document mentionne :

- la date de la première visite de l'agent chargé du contrôle ${ }^{15}$;

- l'existence d'un document intitulé "Charte du cotisant contrôlé », qui présente à la personne contrôlée la procédure de contrôle et les droits dont elle dispose, pendant son déroulement et à son issue. L'avis précise en outre l'adresse électronique où ce document, est consultable et indique qu'il est adressé au cotisant sur sa demande ${ }^{16}$;

- le droit de se faire assister du conseil de son choix ${ }^{17}$;

- la liste des documents demandés (même si celle-ci n'est donnée qu'à titre indicatif).

Notons également que suivant l'article R 243-59 II al. 3, l'inspecteur « peut demander que les documents à consulter lui soient présentés selon un classement nécessaire au contrôle dont il aura au préalable informé la personne contrôlée ».

Cependant, la jurisprudence n'accorde pas au contenu de ce document plus d'importance qu'il n'en faut. Dans un arrêt du 7 mars 2013, la Cour d'appel de Paris a ainsi décidé que «l'avis préalable prévu par l' article R. 243-59 du Code de la sécurité sociale n'a pour objet que d'informer le cotisant de la date de la première visite de l'inspecteur du recouvrement, ainsi que la liste des documents comptables fiscaux et sociaux susceptibles de lui être présentés ${ }^{18}$. Cette position demanderait elle aussi à être nuancée. En effet, s'agissant de la date de première visite, la $2^{\mathrm{e}}$ Chambre civile a statué que l'URSSAF pouvait reporter le contrôle de sa propre initiative. Il lui appartient alors d'en informer en temps utile et par tout moyen approprié l'employeur, et de rapporter la preuve de la réception de I'information en cas de recours contentieux ${ }^{19}$. Quant à la "Charte du cotisant contrôlé »20,

11 Code du travail, art. L 8221-1.

12 Ibid., art L. 8221-5.

13 Cour de cass., ch. civile 2, 21 décembre 2017, n¹7-11535.

14 Voir CSS, art. R 243-59 I al. 2 ; Lettres circulaires ACOSS n¹999-082 du 16 juillet 1999 et $n^{\circ} 2000$ 021 du 17 février 2000.

15 CSS, art. R 243-59 I al. 1.

16 Ibid., art. R 243-59 I al. 5.

17 Ibid., art. R 243-59 II al. 1.

18 Cour d'appel de Paris, pôle 6, ch. 12, 7 mars 2013, n¹0/09376.

19 Cour de cass., ch. civile 2, 15 mars 2018, n¹7-13409.

20 Désormais opposable aux organismes de sécurité sociale depuis le Décret n²016-941 du 8 juillet 2016. 
celle-ci n'est plus remise au cotisant dès le début des opérations de contrôle ${ }^{21}$ et seule la mention du lien internet doit figurer sur l'avis. Enfin, s'agissant de la liste des documents mentionnés sur l'avis, il convient de rappeler qu'elle est indicative et que l'inspecteur peut, au cours du contrôle, exiger la production de pièces complémentaires pouvant être communiquées dans un délai raisonnable, afin d'établir l'application conforme de la législation de sécurité sociale ${ }^{22}$.

Par ailleurs, il n'est pas nécessaire d'indiquer dans l'avis la période contrôlée ${ }^{23}$ et les établissements qui feront l'objet d'une vérification ${ }^{24}$, ou encore que le document soit signé $^{25}$. Autant de restrictions qui limitent la portée de ce document. Pire, si l'on peut admettre qu'un avis de contrôle ne soit pas remis au cotisant en cas de travail dissimulé, on comprend moins pourquoi l'intéressé n'aurait pas connaissance de la Charte du cotisant. Or justement, l'information de cette Charte n'est aujourd'hui communiquée que via l'avis de contrôle. Est-ce à dire que, dans le cadre d'un «travail dissimulé », le cotisant voit ses droits amoindris, alors même que la définition du travail dissimulé est quant à elle particulièrement large ? II s'agit d'une situation ambivalente où l'information sur les droits et devoirs des parties n'est diffusée qu'aux personnes censées être dans la légalité. Il serait probablement nécessaire que, dans ce cadre, la Charte soit remise au cotisant dès le début des opérations de contrôle.

\section{II - UNE LÉGISLATION PERFECTIBLE SUR LA VÉRIFICATION DU CONTRÔLE}

C'est sans aucun doute à ce niveau que les carences sont les plus importantes. Pourtant, face à une législation de plus de plus complexe, il semblerait logique que le principe du contradictoire soit non seulement respecté, mais également développé ${ }^{26}$. De même, il paraît cohérent que les droits des organismes soient clairement définis. Enfin, il serait souhaitable que la sécurité juridique du cotisant, au terme du contrôle, soit assurée. Or, sur ces points essentiels, on peut constater un certain nombre de manquements étonnants dont suivent ici quelques exemples.

Le premier exemple a trait à l'emport de documents par l'inspecteur durant un contrôle sur place voire à la demande de documents par mail ${ }^{27}$. Jusqu'à présent, le Code de la Sécurité sociale était muet sur ces pratiques, même si la doctrine considérait que cette technique se conciliait difficilement avec la notion de vérification « sur place » et avec le respect de la procédure contradictoire. Cette notion d'emport de documents peut revêtir plusieurs aspects tels que la photocopie de documents pour les exploiter directement à l'organisme ou encore l'enregistrement des données comptables sur clé USB pendant le

21 CSS, art. R 243-59 I al. 5 (Décret n²013-1107 du 3 décembre 2013).

22 Cour d'appel de Paris, pôle 6, ch. 13, 30 mars 2018, n¹5/11350.

23 Cour d'appel de Douai, ch. sociale, 31 mai 2018, n¹6/01334.

24 CSS, art. R 243-59 I al. 4.

25 Cour d'appel de Lyon, Sécurité sociale, 16 janvier 2018, n¹6/09070.

26 F. Taquet, "Quelques réflexions sur le respect du principe du contradictoire dans le contrôle URSSAF », Gazette du Palais, n8, 27 février 2018, p. 16.

27 II s'agit ici du contrôle sur place (CSS, art. R 243-59), et non du contrôle sur pièces pour les employeurs occupant moins de 11 salariés (CSS, art. R 243-59-3). 
contrôle ${ }^{28}$. Un arrêt de principe du Conseil d'Etat ${ }^{29}$ a défini, dans le cadre du contrôle fiscal, les trois conditions strictes et cumulatives permettant le déplacement de tout ou partie de la comptabilité des contribuables (sachant que le non-respect de ces conditions entraîne l'irrégularité de la vérification) : certaines formalités doivent être remplies préalablement à l'emport des documents (demande écrite du contribuable et délivrance d'un reçu par le vérificateur), le déplacement des documents comptables ne doit pas avoir pour effet de priver le contribuable du débat oral et contradictoire auquel il a droit, et enfin la restitution des documents doit intervenir avant la clôture des opérations de contrôle.

Il semblerait pourtant pertinent d'étendre ces conditions dans le cadre du contrôle URSSAF. En effet, il pourrait être simplement rappelé que lorsque la vérification est effectuée au sein de l'entreprise, les documents ou supports d'information ne peuvent être emportés par l'inspecteur à l'organisme qu'après autorisation du cotisant. Le récent Décret n²019-1050 du 11 octobre 2019 est venu apporter quelques précisions en la matière. L'article R 243-59 II alinéa 3 du CSS précise ainsi que " sauf autorisation de la personne contrôlée, seules des copies des documents remis peuvent être exploitées hors de ses locaux ». Dès lors, on peut s'interroger sur le fait que ce texte vient légitimer des pratiques récurrentes lors des contrôles. En outre, il convient de signaler que si l'emport de copies de documents peut être fait sans l'accord du cotisant, tel n'est en revanche pas le cas de l'emport des originaux qui nécessite l'accord de l'employeur. D'autre part, les copies de documents doivent avoir été "remises", c'est-à-dire confiées par le cotisant (et non prises de force). Par ailleurs, relevons que dans tous les cas, la procédure contradictoire (c'est-à-dire un échange sur le contenu de ces documents) devra avoir été respectée. Enfin, il est clair qu'en cas de contentieux relatif à un éventuel emport de document en dehors du cadre légal, il appartiendrait au cotisant d'apporter la preuve de ses affirmations.

S'agissant de la demande de documents par mail, elle est relativement tentante pour l'inspecteur qui peut ainsi achever son contrôle sur place plus rapidement. Certes, les dispositions de l'article R 243-59-1 du CSS prévoit expressément les conditions de vérification de documents dématérialisés. Mais, dans la pratique, nombre d'URSSAF n'hésitent pas à réclamer des documents par mail en dehors de tout respect de la procédure contradictoire. Sans doute serait-il nécessaire de rappeler solennellement les devoirs des organismes en la matière ${ }^{30}$.

Un autre exemple concerne la procédure contradictoire suite à la vérification. Actuellement, dès lors qu'un organisme de recouvrement effectue un redressement, il fait parvenir au cotisant un courrier d'observations auquel l'intéressé peut répondre dans un

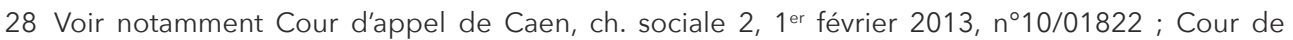
Cass., ch. civile 2, 11 juillet 2013, n¹2-17940 ; Cour d'appel de Paris, pôle 6, ch. 12, 6 juillet 2017, $n^{\circ} 15 / 12849$.

29 Conseil d'Etat, sect., 21 mai 1976, n 94052.

30 Voir Cour d'appel de Paris, pôle 6, ch. 12, 15 février 2019, n¹4/12280. 
délai de 30 jours $^{31}$. Lorsque le cotisant a répondu, l'inspecteur doit obligatoirement formuler une réponse avant que ne soit envoyée une mise en demeure. Cependant, la procédure issue du Décret n²007-546 du 11 avril 2007 était imparfaite dans la mesure où le texte ne précisait pas le type de réponse devant être apporté, et ne créait pas de véritable dialogue entre le cotisant et I'URSSAF. Le Décret du 8 juillet 2016 a partiellement répondu à cette critique, en énonçant que chaque observation exprimée de manière circonstanciée devait faire l'objet d'une réponse motivée ${ }^{32}$. Toutefois, les pouvoirs publics ne sont pas réellement allés jusqu'au bout de leur démarche puisque le cotisant va répondre au même inspecteur qui a effectué le contrôle, étant peu probable que ce dernier change d'avis sauf production d'éléments nouveaux. Au final, on décèle ici les limites de la procédure contradictoire qui se révèle bien souvent en la matière comme étant une simple obligation de façade ${ }^{33}$. En l'occurrence, le cotisant devrait disposer de la possibilité de saisir un autre interlocuteur, voire même un médiateur.

Citons enfin l'exemple relatif à la décision implicite d'accord. L'article R 243-59 alinéa 5 du CSS, issu du Décret n99-434 du 28 mai 1999, mentionnait qu'« à l'issue du contrôle, les inspecteurs du recouvrement communiquent à l'employeur ou au travailleur indépendant un document daté et signé par eux mentionnant l'objet du contrôle, les documents consultés, la période vérifiée et la date de la fin du contrôle ». L'intérêt de cette mention apparaissait à la lecture de l'article R 243-59 dernier alinéa du CSS, suivant lequel l'absence d'observations des inspecteurs lors d'une vérification « vaut accord tacite concernant les pratiques ayant donné lieu à vérification, dès lors que l'organisme de recouvrement a eu les moyens de se prononcer en toute connaissance de cause. Le redressement ne peut porter sur des éléments qui, ayant fait l'objet d'un précédent contrôle dans la même entreprise ou le même établissement, n'ont pas donné lieu à observations de la part de cet organisme ». Or, cette disposition qui s'inspirait du droit fiscal était souhaitée par les cotisants, notamment pour garantir une plus grande sécurité juridique. Toutefois, les organismes de sécurité sociale ${ }^{34}$ avaient fait une interprétation restrictive de ces dispositions, soutenant qu'il appartenait à l'employeur d'apporter la preuve de l'accord tacite de l'inspecteur, et ajoutant que d'autres éléments devaient être cumulativement réunis, notamment l'absence d'observation par l'organisme du recouvrement sur des pratiques vérifiées lors du précédent contrôle, la preuve que le contrôleur avait examiné les points litigieux et que

31 Cette rapidité de la procédure était souhaitée par les rédacteurs du Code de la Sécurité sociale. Le délai de réponse était à l'origine de 8 jours, porté à 15 jours par le Décret n90-1009 du 14 novembre 1990, puis à 30 jours par le Décret n99-434 du 28 mai 1999. Ce délai de 30 jours peut être prolongé, à la demande de la personne contrôlée, à 60 jours. A défaut de réponse de l'organisme de recouvrement, la prolongation du délai est considérée comme étant acceptée (CSS, art. L 243-7-1 A - R 243-59 III). La réponse du cotisant ne constitue pas une obligation, et l'absence de réponse ne saurait être assimilée à une approbation du redressement effectué (voir notamment Cour de Cass., ch. civile 2, 9 février 2006, $\mathrm{n}^{\circ} 04-30.535$ ).

32 Voir notamment Cour de cass., ch. civile 2, 12 mars 2015, n¹4-16019; Cour d'appel de Paris, pôle 6, ch. 12, 27 novembre 2014, nº12/11321.

33 L'administration fiscale l'a bien compris puisque la Charte du contribuable (opposable à l'administration) énonce: " Si le vérificateur a maintenu totalement ou partiellement les redressements envisagés, des éclaircissements supplémentaires peuvent vous être fournis si nécessaire par l'inspecteur (...) [et] si, après ces contacts, des divergences importantes subsistent, vous pouvez faire appel à l'interlocuteur départemental qui est un fonctionnaire de rang élevé spécialement désigné par le directeur dont dépend le vérificateur ».

34 Lettre circulaire ACOSS n²000-21 du 17 février 2000. 
tous les éléments nécessaires à son information lui avaient été transmis. La jurisprudence avait pris la même position.

En pratique, pour se prévaloir d'un accord implicite, le cotisant devait apporter la preuve que le silence de l'organisme s'accompagnait de circonstances permettant de déduire que l'inspecteur avait pris position en connaissance de cause ${ }^{35}$, qu'il portait sur une situation identique à celle ayant fait l'objet d'une décision implicite lors de précédents contrôles ${ }^{36}$, et enfin que ce silence concernait des éléments qui avaient fait l'objet d'un précédent contrôle dans la même entreprise ou le même établissement (non dans d'autres sociétés du même groupe $)^{37}$. Dans tous les cas, la charge de la preuve de l'existence d'une décision implicite incombait à l'employeur ${ }^{38}$. Malgré les critiques de la doctrine, les nouvelles dispositions introduites par le Décret du 8 juillet 2016 ne modifient pas fondamentalement cette situation. En effet, l'article R 243-59-7 du CSS prévoit que la vérification doit avoir porté sur des éléments ayant fait l'objet d'un précédent contrôle dans la même entreprise ou le même établissement ${ }^{39}$, que l'organisme a pu se prononcer en toute connaissance de cause, que les circonstances de droit et de fait au regard desquelles les éléments ont été examinés sont inchangées ${ }^{40}$.

Cette absence d'audace des pouvoirs publics pourrait surprendre. En effet, les organismes de sécurité sociale sont tenus vis-à-vis des usagers à une obligation $d^{\prime}$ information et de conseil ${ }^{41}$ et chacun sait que, dans le " maquis législatif » aujourd'hui constitué par le droit de la sécurité sociale, le conseil, l'information et la sécurité juridique ne sont pas des vains mots.

\section{III - UNE LÉGISLATION PERFECTIBLE SUR LE CONTENTIEUX}

Le contentieux est divisé en deux étapes : une étape "administrative » devant les Commissions de recours amiable (ci-après dénommées CRA) des organismes de recouvrement ${ }^{42}$, et une étape judiciaire. Ces Commissions, qui portaient avant le Décret n86-58 du 18 mars 1986 le nom de "Commissions de recours gracieux », ont été créées après la seconde guerre mondiale ${ }^{43}$. A cet égard, la lecture de l'exposé des motifs de la Loi du 24 octobre 1946 est particulièrement intéressant : " II est apparu utile que les

35 Cour de cass., ch. sociale, 5 juin 1997, n²95-17763.

36 Cour de cass., ch. sociale, 16 avril 1992, n89-16089.

37 Cour de cass., ch. civile 2, 8 juill. 2010, n09-15782.

38 Cour de Cass., ch. sociale, 11 avril 2002, $n^{\circ} 00-15730$.

39 C'est au cotisant qu'incombe la preuve, laquelle ne peut porter que sur la base des documents consultés et mentionnés par I'URSSAF (CSS, art. R 243-59 III al. 1).

40 Voir Cour de cass., ch. civile 2, 20 décembre 2018, n¹7-26952. La proposition n¹0 du Rapport parlementaire Gérard-Goua (op. cit.) préconisait que l'absence d'observations lors d'un contrôle puisse valoir accord tacite concernant les pratiques mises en œuvre par l'entreprise, à moins que le cotisant n'ait pas agi de bonne foi ou ait fourni des éléments incomplets ou inexacts.

41 CSS, art. R 112-2.

42 F. Taquet, "Faut-il supprimer la commission de recours amiable dans le contentieux URSSAF ? ", JCP, Ed. E, 2009, nº1801.

43 Si à la fin de la seconde guerre mondiale, les cotisations sociales étaient peu élevées et la législation de la sécurité sociale simple, peu sujette à contestation, tel n'est plus le cas aujourd'hui dans notre pays où la loi « change à chaque saison ». Dès lors, le rôle de CRA devrait être revu. 
réclamations soient, avant l'intervention de toute juridiction, portées devant le conseil d'administration de l'organisme compétent, de manière que le cas soit examiné, non pas seulement par un employé irresponsable de la Caisse, mais par les administrateurs eux-mêmes, c'est à dire en fait par les représentants des intéressés " ${ }^{44}$. Le législateur considérait donc cette Commission - émanation du conseil d'administration de chaque Caisse - comme un filtre chargé de procéder à un nouvel examen des dossiers avant la phase judiciaire ${ }^{45}$. L'appréciation portée sur ces Commissions diffère selon le type d'organismes concerné (CPAM - CARSAT - CAF). En effet, dans ces dernières entités, les CRA jouent un rôle social et humain qui ne doit pas être sous-estimé. En revanche, le rôle de ces entités semble beaucoup plus discuté dès lors qu'une contestation apparaît sur le fond d'un dossier, et qu'un enjeu financier est en cause ${ }^{46}$. Le Rapport parlementaire Fouquet précité le relevait, estimant que : «La procédure suivie est imparfaite et respecte peu les exigences du contradictoire $»^{47}$.

Les derniers Rapports avaient proposé d'importantes modifications en matière d'ouverture de ces Commissions et de formation des administrateurs ${ }^{48}$. Là encore, la transparence et l'audace ont fait défaut en renvoyant une réforme ultérieure. Pire, il semblerait même que la Loi n²018-727 du 10 août 2018 (généralisant notamment la médiation au sein des organismes de sécurité sociale), ait davantage compliqué le système ${ }^{49}$. Certes, la médiation ne constitue qu'une possibilité50, une faculté complémentaire dont dispose le cotisant ${ }^{51}$. Toutefois, on peut cependant se demander si, à l'heure où le rôle des

44 A. de Cambourg, «La procédure gracieuse préalable dans les contestations de la Sécurité sociale», Droit social, 1954, p. 639.

45 La CRA n'est pas une juridiction mais une émanation du conseil d'administration de l'organisme de sécurité sociale concerné, et n'est pas, de ce fait, soumise aux dispositions de la Convention européenne des droits de l'homme (Cour de cass., ch. civile 2, 8 novembre 2006, n05-14075).

46 Voir «Les recours amiables devant I'URSSAF», Droit social, 1987, p. 560. G. Pigaglio, ancien directeur de I'URSSAF de Lyon, note que la masse des dossiers retenus en une seule séance rend illusoire l'examen de chacun d'eux, et ce d'autant que les administrateurs présents n'ont pas toujours la compétence indispensable pour porter un avis éclairé: "sans un effort de formation important et continu de la part des organisations syndicales ouvrières ou patronales, l'administrateur risque de s'en remettre souvent à la compétence des services administratifs et de ne plus (...) jouer valablement son rôle ". Voir également H.-G. Bascou et J.-C. Ranc : "La Commission de Recours Amiable est trop souvent soumise aux pouvoirs de I'URSSAF et les administrateurs ont beaucoup de mal à prendre des décisions contraires à celles des agents chargés du contrôle », op. cit.

47 Rapport parlementaire Fouquet, op. cit., p. 37.

48 Voir notamment les propositions n44, 45 et 46 du Rapport Fouquet (op. cit.) et n³9, 40, 41 du Rapport Gérard-Goua (op. cit.).

49 Art. 34 de la Loi n²018-727 du 10 août 2018; CSS, art. L. 271-7-1.

50 Les réclamations "peuvent être présentées " sans préjudice des voies de recours existantes, devant le médiateur de l'organisme concerné. Inversement, les dispositions de l'art. R 142-1 du CSS prévoient que les réclamations formées contre les décisions prises par les organismes de Sécurité sociale et de Mutualité sociale agricole de salariés ou de non-salariés «sont soumises »à une CRA composée et constituée au sein du conseil d'administration de chaque organisme. Aussi, le cotisant qui souhaite contester le redressement opéré par I'URSSAF doit préalablement saisir la CRA. Il s'agit là d'une disposition d'ordre public (voir notamment Cour de cass., ch. civile, 20 juin 1958. Bull. civ. II, n454; Cour d'appel de Paris, pôle 6, ch. 12, 30 mars 2018, n¹4/09633).

51 Suivant l'art. L. 217-7-1 I al. 2 du CSS, le médiateur exerce ses fonctions " en toute impartialité », mais le fait qu'il soit dans le même alinéa « désigné par le directeur de l'organisme » peut susciter quelques interrogations. 


\section{LA PROCÉDURE DE CONTRÔLE URSSAF}

Commissions de recours amiables est souvent décrié, cet empilement de procédures était nécessaire.

Pour conclure, force est de constater que les textes relatifs à la procédure de contrôle sont pour le moins perfectibles. II serait temps de créer un cadre qui donne aux cotisants des garanties réelles, qui incite au dialogue et promeut la sécurité juridique. Tout le monde y gagnerait, non seulement les cotisants, mais aussi les organismes de recouvrement qui savent pertinemment que sans l'adhésion et la confiance des entreprises (lesquelles financent à $90 \%$ la protection sociale), toute réforme reste vouée à l'échec.

\section{FRANÇOIS TAQUET}

Avocat spécialiste en Droit du travail et protection sociale, Professeur de Droit social (IESEG, Skema BS), Directeur scientifique du réseau international d'avocats GESICA.

Thèmes de recherche : Sécurité sociale (procédures de contrôle et de recouvrement), ruptures conventionnelles individuelles et collectives du contrat de travail, assurance chômage.

\section{Publications:}

F. Taquet, « El derecho laboral frances alconzado por la reforma. Como se adapta Francia al entorno europeo ", Revista RYD Republica y derecho, Université Nacionale de Cuyo, Mendoza (Argentine), Septembre 2018.

F. Taquet, Contrôle URSSAF : Entre droits et obligations, Editions Gereso, 2018, 261 pages. 